\title{
Hybrid technique for MHD free convection flow and heat transfer of Nano-fluid
}

\author{
G.BalReddy ${ }^{1}$ \\ ${ }^{1}$ Assistant Professor, Mahatma Gandhi Institute of Technology, Hyderabad, Telangana.
}

\begin{abstract}
A numerical model is created for the nanofluid stream and warmth move because of the imprudent movement of an endless vertical permeable plate in its own plane within the sight of an attractive field and gooey dissemination. The overseeing flimsy, coupled, nonlinear incomplete differential conditions are changed into an arrangement of nonlinear standard differential conditions, with suitable limit conditions. A powerful Galerkin limited component mathematical arrangement is created. A [1]scope of nanofluids containing nanoparticles of aluminum oxide, copper, titanium oxide and silver with nanoparticle volume division goes not exactly or equivalent to 0.04 are thought of. The Tiwari-Das nanofluid model is utilized. The [2]speed and temperature profiles just as the skin grating coefficient and Nusselt number are inspected for various boundaries, for example, nanoparticle volume part, nanofluid type, attractive boundary, warm Grashof number, Eckert number and pull boundary. The current reproductions are applicable to attractive nanomaterials warm stream preparing in the synthetic designing and metallurgy ventures.
\end{abstract}

Keywords—Nanofluid, Galerkin, copper, numerical model ,metallurgy.

\section{INTRODUCTION}

\begin{tabular}{|ll|}
\hline Nomenclature \\
$\mathrm{M}$ & Magnetic parameter \\
$\mathrm{Ec}$ & Eckert number \\
$\lambda$ & suction parameter \\
$\mathrm{Gr}$ & thermal Grashof number \\
$\mathrm{t}$ & time \\
$\mathrm{T}$ & temperature \\
$\mathrm{U}$ & velocity along x-axis, \\
$\rho$ & density \\
$\mu$ & dynamic viscosity \\
$\beta$ & thermal expansion coefficient \\
$\mathrm{g}$ & acceleration due to gravity, \\
$\kappa$ & thermal conductivity \\
$\mathrm{n}$ & empirical shape factor for the nanoparticle \\
$\phi$ & nanoparticle volume fraction \\
$V$ & kinematic viscosity \\
subscripts \\
$\mathrm{nf}$ & nanofluids \\
$\mathrm{f}$ & base fluid \\
$\mathrm{s}$ & solid nanoparticles \\
\hline
\end{tabular}

Convectional heat move liquids, including oil, water, and ethylene glycol blend are helpless warmth move fluids. Since [3] the warm conductivity of these liquids assumes a significant part in deciding the coefficient of warmth move between the warmth move medium and the warmth move surface, various techniques [4] have been utilized to improve the warm conductivity of these liquids by suspending nanometre/micrometer-sized molecule materials in liquids. Intensive consideration has been aimed at mathematical reproductions of normal convection heat move in nanofluids both with and without attractive fields, and delegate examines incorporate [5]Congedo et al.(2009). Uddin et al.(2014) utilized MAPLE emblematic quadrature to concentrate mathematically the electromagnetic nanofluid stream in permeable media with divider slip [6] and radiation heat move impacts. Bég et al.(2014) applied understood limited contrast techniques to dissect the attractive bio-nanofluid acceptance stream from an extending surface with surface strain impacts. Rana et al. (2013) utilized a variation limited component [7]technique to mimic pivoting attractive nanofluid limit layer stream, warmth and mass exchange from an expelling sheet. As of late Rajesh et al.(2014) introduced a numerical model for the flimsy free convective stream and warmth move of a thick nanofluid from a moving vertical chamber within the sight of warm radiation. Later Rajesh and Anwar Beg (2014) mathematically examined the impacts of MHD on the transient free convection stream of a gooey, electrically leading, and incompressible nanofluid past a moving semi-limitless 


\section{International Advanced Research Journal in Science, Engineering and Technology}

Vol. 8, Issue 6, June 2021

DOI: $10.17148 /$ IARJSET.2021.86124

vertical chamber with temperature oscillation. Transient free [8]convection streams affected by an attractive field have drawn in light of a legitimate concern for some specialists considering their applications in present day materials preparing where attractive fields are known to accomplish great control and control of electrically-directing materials (Ibrahim and Shankar, 014).Magneto hydrodynamic (MHD) convection streams likewise discover huge applications in environmentally friendly power devices, including MHD power generators (Chen et. al.2005, Yamaguchi, 2011) just as atomic reactor transport measures (Mukhopadhyay, 2011) wherein attractive field is utilized to direct warmth move rates. Taking into account these applications, [9]the current investigation is proposed to analyze the impacts of cross over attractive field on transient free convective progression of nanofluid past an imprudently begun limitless vertical permeable plate with thick scattering. The [10]present investigation additionally gives a significant benchmark to additional re-enactments of magneto-nanofluid dynamic vehicle marvels of importance to materials preparing, with elective computational calculations.

\section{EXPERIMENTAL METHODS OR METHODOLOGY:}

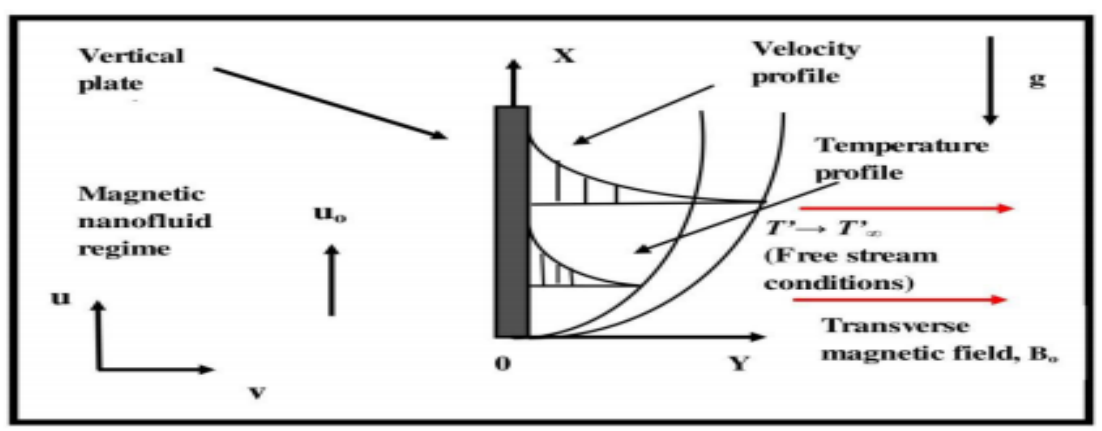

Figure 1: The physical model and coordinate system

$$
\begin{aligned}
& \frac{\partial v}{\partial y}=0 \\
& \frac{\partial u}{\partial t^{\prime}}+v \frac{\partial u}{\partial y}=v_{n f} \frac{\partial^{2} u}{\partial y^{2}}+\frac{(\rho \beta)_{n f}}{\rho_{n f}} g\left(T^{\prime}-T_{\infty}^{\prime}\right)-\frac{\sigma B_{0}^{2} u}{\rho_{u f}}
\end{aligned}
$$


$\frac{\partial T^{\prime}}{\partial t^{\prime}}+v \frac{\partial T^{\prime}}{\partial y}=\frac{\kappa_{n f}}{\left(\rho c_{p}\right)_{n f}} \frac{\partial^{2} T^{\prime}}{\partial y^{2}}+\frac{\mu_{n f}}{\left(\rho c_{p}\right)_{n f}}\left(\frac{\partial u}{\partial y}\right)^{2}$

The initial and boundary conditions are

$$
\begin{array}{rccc}
t^{\prime} \leq 0: \mathrm{u}=0, & \mathrm{~T}^{\prime}=\mathrm{T}_{\infty}^{\prime} & \text { for all } \mathrm{y} \\
t^{\prime}>0: \mathrm{u}=\mathrm{u}_{0}, & \mathrm{~T}^{\prime}=\mathrm{T}_{w}^{\prime} & \text { for } & \mathrm{y}=0 \\
\mathrm{u} \rightarrow 0, & \mathrm{~T}^{\prime} \rightarrow \mathrm{T}_{\infty}^{\prime} & \text { as } & \mathrm{y} \rightarrow \infty
\end{array}
$$

Equation (1) gives $\quad v=-v_{0}\left(v_{0}>0\right)$

Where $v_{0}$ is the constant suction velocity and the negative sign indicates that it is towards the plate.

For nanofluids, the expressions of density $\rho_{n f}$, thermal expansion coeffcient $(\rho \beta)_{n f}$ and heat capacitance $\left(\rho c_{p}\right)_{n f}$ are given by

$\rho_{n f}=(1-\phi) \rho_{f}+\phi \rho_{s}$

$(\rho \beta)_{n f}=(1-\phi)(\rho \beta)_{f}+\phi(\rho \beta)_{s}$

$\left(\rho C_{p}\right)_{n f}=(1-\phi)\left(\rho C_{p}\right)_{f}+\phi\left(\rho C_{p}\right)_{s}$

The effective thermal conductivity of the nanofluid according to Hamilton and Crosser (1962) model is given by

$$
\frac{\kappa_{e f f}}{\kappa_{f}}=\frac{\kappa_{s}+(n-1) \kappa_{f}-(n-1) \phi\left(\kappa_{f}-\kappa_{s}\right)}{\kappa_{s}+(n-1) \kappa_{f}+\phi\left(\kappa_{f}-\kappa_{s}\right)}
$$

Where $\mathrm{n}$ is the empirical shape factor for the nanoparticle. In particular, $\mathrm{n}=3$ for spherical shaped nanoparticles and $\mathrm{n}=3 / 2$ for cylindrical ones.

Table 1. Thermo-physical properties of water and nanoparticles

\begin{tabular}{llllll}
\hline & $\mathrm{H}_{2} \mathrm{O}$ & $\mathrm{Al}_{2} \mathrm{O}_{3}$ & $\mathrm{Cu}$ & $\mathrm{TiO}_{2}$ & $\mathrm{Ag}$ \\
\hline$\rho\left(\mathrm{Kg} \mathrm{m}^{-3}\right)$ & 997.1 & 3970 & 8933 & 4250 & 10500 \\
$\mathrm{C}_{p}\left(\mathrm{~J} \mathrm{Kg}^{-1} \mathrm{~K}^{-1}\right)$ & 4179 & 765 & 385 & 686.2 & 235 \\
$\kappa\left(\mathrm{Wm}^{-1} \mathrm{~K}^{-1}\right)$ & 0.613 & 40 & 401 & 8.9528 & 429 \\
$\beta \times 10^{-5}\left(\mathrm{~K}^{-1}\right)$ & 21 & 0.85 & 1.67 & 0.9 & 1.89 \\
\hline
\end{tabular}

Introducing the following non dimensional variables in equations (2) and (3):

$$
U=\frac{u}{u_{0}}, t=\frac{t^{\prime} u_{0}^{2}}{v_{f}}, Y=\frac{y u_{0}}{v_{f}}, T=\frac{T^{\prime}-T_{\infty}^{\prime}}{T_{w}^{\prime}-T_{\infty}^{\prime}}, G_{r}=\frac{g \beta_{f} v_{f}\left(T_{w}^{\prime}-T_{\infty}^{\prime}\right)}{u_{0}^{3}}, P_{r}=\frac{v_{f}}{\alpha_{f}}=\frac{\left(\mu c_{p}\right)_{f}}{k_{f}},
$$




$$
M=\frac{\sigma B_{0}^{2} v_{f}}{\rho_{f} u_{0}^{2}}, \lambda=-\frac{v}{u_{0}}, E_{c}=\frac{u_{0}^{2}}{\left(c_{p}\right)_{f}\left(T_{w}^{\prime}-T_{\infty}^{\prime}\right)}
$$

The governing equations reduces to

$$
\frac{\partial U}{\partial t}-\lambda \frac{\partial U}{\partial Y}=\frac{1}{(1-\phi)^{2.5}} \frac{1}{\left(1-\phi+\phi\left(\frac{\rho_{s}}{\rho_{f}}\right)\right)} \frac{\partial^{2} U}{\partial Y^{2}}+\frac{\left(1-\phi+\phi \frac{(\rho \beta)_{s}}{(\rho \beta)_{f}}\right)}{\left(1-\phi+\phi \frac{(\rho)_{s}}{(\rho)_{f}}\right)} G r T-\frac{1}{\left(1-\phi+\phi\left(\frac{\rho_{s}}{\rho_{f}}\right)\right)} M U
$$

$$
\frac{\partial T}{\partial t}-\lambda \frac{\partial T}{\partial Y}=\frac{k_{n f}}{k_{f}} \frac{1}{\left(1-\phi+\phi \frac{\left(\rho c_{p}\right)_{s}}{\left(\rho c_{p}\right)_{f}}\right)} \frac{1}{P_{r}} \frac{\partial^{2} T}{\partial Y^{2}}+\frac{1}{(1-\phi)^{2.5}} \frac{1}{\left(1-\phi+\phi \frac{\left(\rho c_{p}\right)_{s}}{\left(\rho c_{p}\right)_{f}}\right)} E_{c}\left(\frac{\partial U}{\partial Y}\right)^{2}
$$

The corresponding dimensionless boundary conditions are:

$$
\begin{array}{rrrc}
t \leq 0: \mathrm{U}=0, & T=0 & \text { for all } & \mathrm{Y} \\
t>0: \mathrm{U}=1, & T=1 & \text { at } & \mathrm{Y}=0 \\
\mathrm{U} \rightarrow 0, & T \rightarrow 0 & \text { as } & \mathrm{Y} \rightarrow \infty
\end{array}
$$

Let $E_{1}=\frac{1}{(1-\phi)^{2.5}} \frac{1}{\left(1-\phi+\phi\left(\frac{\rho_{s}}{\rho_{f}}\right)\right)}, \quad E_{2}=\frac{\left(1-\phi+\phi \frac{(\rho \beta)_{s}}{(\rho \beta)_{f}}\right)}{\left(1-\phi+\phi \frac{(\rho)_{s}}{(\rho)_{f}}\right)}, E_{3}=\frac{1}{\left(1-\phi+\phi\left(\frac{\rho_{s}}{\rho_{f}}\right)\right)}$,

$$
E_{4}=\frac{k_{n f}}{k_{f}} \frac{1}{\left(1-\phi+\phi \frac{\left(\rho c_{p}\right)_{s}}{\left(\rho c_{p}\right)_{f}}\right)}, E_{5}=\frac{1}{(1-\phi)^{2.5}} \frac{1}{\left(1-\phi+\phi \frac{\left(\rho c_{p}\right)_{s}}{\left(\rho c_{p}\right)_{f}}\right)}
$$

Now the equations (8) and (10) in terms of $E_{1}, E_{2}, E_{3}, E_{4}$ and $E_{5}$ are

$$
\begin{aligned}
& \frac{\partial U}{\partial t}-\lambda \frac{\partial U}{\partial Y}=E_{1} \frac{\partial^{2} U}{\partial Y^{2}}+E_{2} G r T-E_{3} M U \\
& \frac{\partial T}{\partial t}-\lambda \frac{\partial T}{\partial Y}=E_{4} \frac{1}{P_{r}} \frac{\partial^{2} T}{\partial Y^{2}}+E_{c} E_{5}\left(\frac{\partial U}{\partial Y}\right)^{2}
\end{aligned}
$$




\section{SOLUTION}

$$
\begin{aligned}
& \int_{Y_{j}}^{Y_{k}} N^{(e)^{T}}\left(E_{1} \frac{\partial^{2} U^{(e)}}{\partial Y^{2}}+\lambda \frac{\partial U^{(e)}}{\partial Y}-\frac{\partial U^{(e)}}{\partial t}+E_{2} G r T-E_{3} M U^{(e)}\right) d Y=0 \\
& \int_{Y_{j}}^{Y_{k}} N^{(e)^{T}}\left(E_{4} \frac{1}{\operatorname{Pr}} \frac{\partial^{2} T^{(e)}}{\partial Y^{2}}+\lambda \frac{\partial T^{(e)}}{\partial Y}-\frac{\partial T^{(e)}}{\partial t}+E_{c} E_{5}\left(\frac{\partial U}{\partial Y}\right)^{2}\right) d Y=0
\end{aligned}
$$

Let the linear, piecewise approximation solution be

$$
\begin{aligned}
& U^{e}=N_{j}(Y) U_{j}(t)+N_{k}(Y) U_{k}(t)=N_{j} U_{j}+N_{k} U_{k} \\
& T^{e}=N_{j}(Y) T_{j}(t)+N_{k}(Y) T_{k}(t)=N_{j} T_{j}+N_{k} T_{k}
\end{aligned}
$$

Where

$$
N_{j}=\frac{Y_{k}-Y}{Y_{k}-Y_{j}}, N_{k}=\frac{Y-Y_{j}}{Y_{k}-Y_{j}}, N^{(e)^{T}}=\left[\begin{array}{ll}
N_{j} & N_{k}
\end{array}\right]^{T}=\left[\begin{array}{c}
N_{j} \\
N_{k}
\end{array}\right]
$$

Table 2. Thermal conductivity and dynamic viscosity for spherical shaped nanoparticles

\begin{tabular}{cccc}
\hline Model & Shape of nanoparticles & Thermal conductivity & Dynamic viscosity \\
\hline I & Spherical & $\frac{\kappa_{n f}}{\kappa_{f}}=\frac{\kappa_{s}+2 \kappa_{f}-2 \phi\left(\kappa_{f}-\kappa_{s}\right)}{\kappa_{s}+2 \kappa_{f}+\phi\left(\kappa_{f}-\kappa_{s}\right)}$ & $\mu_{n f}=\frac{\mu_{f}}{(1-\phi)^{2.5}}$ \\
II & Spherical & $\frac{\kappa_{n f}}{\kappa_{f}}=\frac{\kappa_{s}+2 \kappa_{f}-2 \phi\left(\kappa_{f}-\kappa_{s}\right)}{\kappa_{s}+2 \kappa_{f}+\phi\left(\kappa_{f}-\kappa_{s}\right)}$ & $\mu_{n f}=\mu_{f}\left(1+7.3 \phi+123 \phi^{2}\right)$ \\
\hline
\end{tabular}

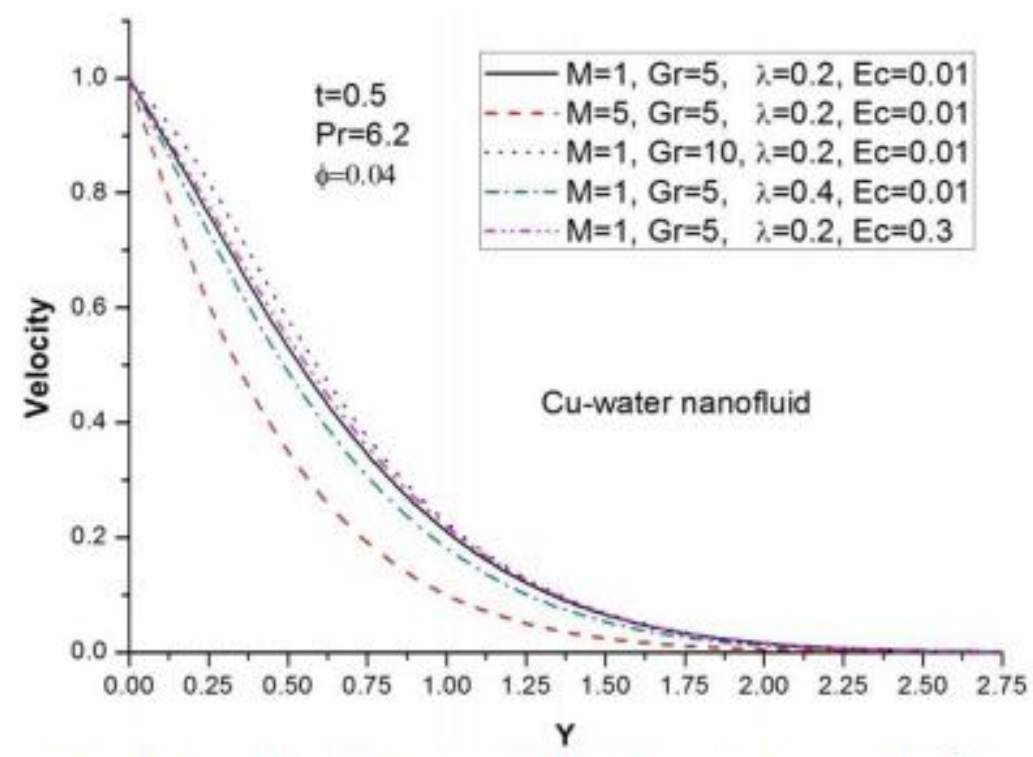

Figure 2. Transient velocity profiles for different $\mathbf{M}, \mathbf{G r}, \mathbf{E c}, \lambda$ 
International Advanced Research Journal in Science, Engineering and Technology

Vol. 8, Issue 6, June 2021

DOI: $10.17148 / I A R J S E T .2021 .86124$

4. RESULTS AND DISCUSSIONS:

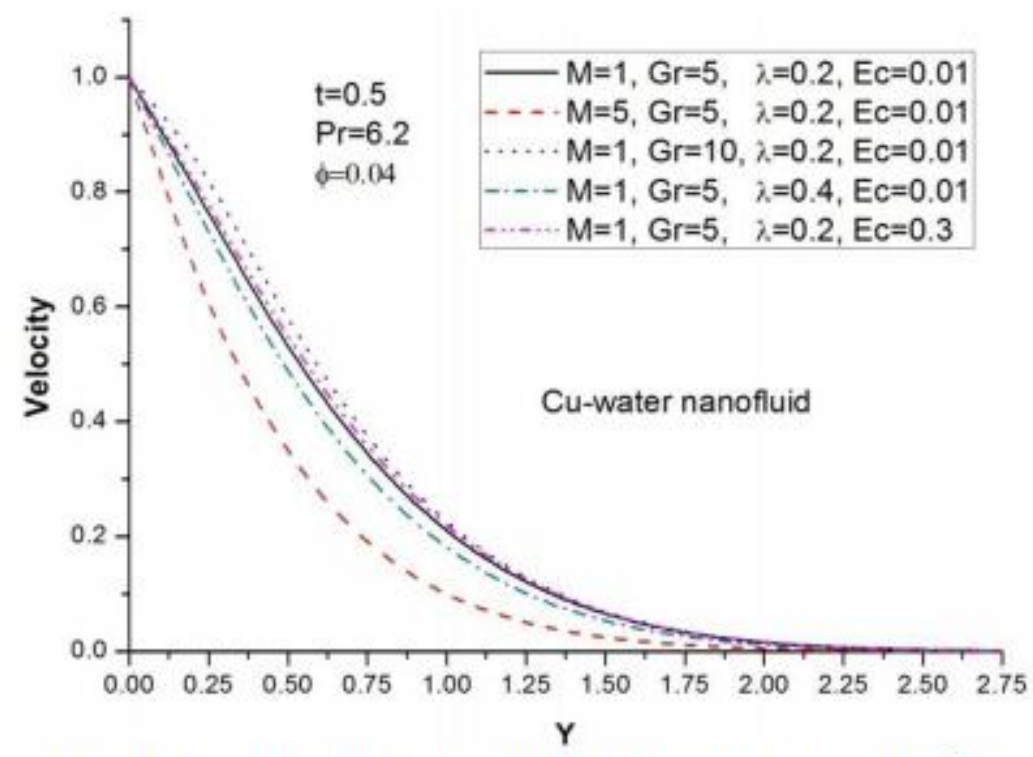

Figure 2. Transient velocity profiles for different $\mathrm{M}, \mathrm{Gr}, \mathrm{Ec}, \lambda$

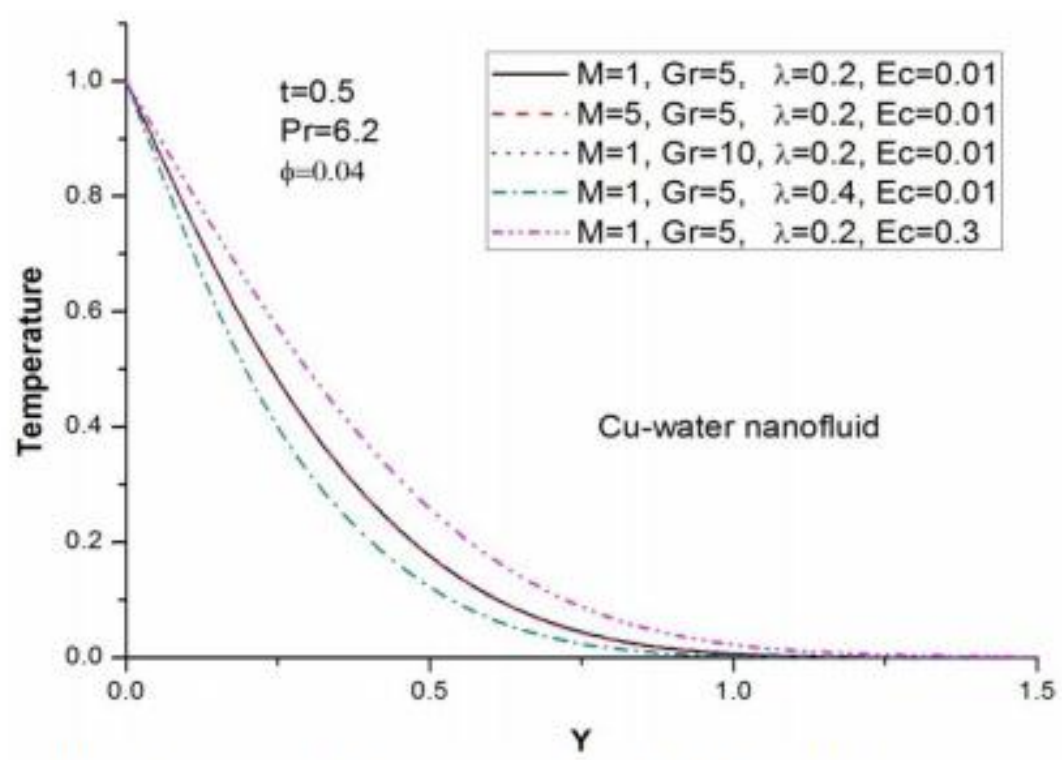

Figure 3. Transient temperature profiles for different $\mathbf{M}, \mathbf{G r}, \mathbf{E c}, \lambda$ 
International Advanced Research Journal in Science, Engineering and Technology

Vol. 8, Issue 6, June 2021

DOI: $10.17148 / I A R J S E T .2021 .86124$

Table 3. Transient velocity of $\mathrm{Cu}$-water nanofluid for different $\phi$ and $\mathbf{t}$

\begin{tabular}{rrrrrrr}
\hline & \multicolumn{3}{c}{$\mathrm{t}=0.5$} & & \multicolumn{3}{c}{$\mathrm{t}=1$} \\
\hline $\mathrm{Y}$ & $\phi=0$ & $\phi=0.02$ & $\phi=0.04$ & $\phi=0$ & $\phi=0.02$ & $\phi=0.04$ \\
\hline 0 & 1 & 1 & 1 & 1 & 1 & 1 \\
0.4 & 0.6376 & 0.6286 & 0.6209 & 0.7288 & 0.7252 & 0.7219 \\
0.8 & 0.3391 & 0.3256 & 0.3145 & 0.4587 & 0.4533 & 0.4486 \\
1 & 0.2363 & 0.2224 & 0.211 & 0.3521 & 0.3456 & 0.3401 \\
1.4 & 0.1056 & 0.0941 & 0.0851 & 0.1987 & 0.191 & 0.1845 \\
1.8 & 0.0417 & 0.0346 & 0.0293 & 0.107 & 0.0997 & 0.0937 \\
2 & 0.0249 & 0.0197 & 0.0161 & 0.0772 & 0.0705 & 0.0651 \\
2.4 & 0.0079 & 0.0057 & 0.0042 & 0.0386 & 0.0337 & 0.0299 \\
2.8 & 0.0022 & 0.0013 & 0.0009 & 0.0182 & 0.0151 & 0.0127 \\
3 & 0.0011 & 0.0006 & 0.0004 & 0.0122 & 0.0098 & 0.0081 \\
3.4 & 0.0002 & 0.0001 & 0.0001 & 0.0053 & 0.0039 & 0.003 \\
3.8 & 0 & 0 & 0 & 0.0021 & 0.0014 & 0.001 \\
4 & 0 & 0 & 0 & 0.0013 & 0.0009 & 0.0006 \\
\hline
\end{tabular}

\section{CONCLUSION}

This paper explored transient MHD free convection stream and warmth move of nanofluid past an imprudently begun vertical permeable plate within the sight of gooey scattering. Mathematical computations are done for different upsides of the dimensionless boundaries. The impacts of different actual boundaries on the nanofluid stream and warmth move attributes were inspected. The current calculations have shown that the pace of warmth move diminished with the expansion in Magnetic boundary and Eckert number. In any case, as Suction boundary and nanoparticle volume part expanded, the pace of warmth move expanded for all nanofluids. Picking copper as nanoparticle prompts the improved warmth move rate contrasted with the other nanofluids.

\section{REFERENCES}

1. Matthew, A. R., Prasad, A., \& Anto, P. B. (2017, July). A review on feature extraction techniques for tumor detection and classification from brain MRI. In 2017 International Conference on Intelligent Computing, Instrumentation and Control Technologies (ICICICT) (pp. 1766-1771). IEEE.

2. Kharat, K. D., Kulkarni, P. P., \& Nagori, M. B. (2012). Brain tumor classification using neural network based methods. International Journal of Computer Science and Informatics, 1(4), 2231-5292.

3. Dhanalakshmi, P., \& Kanimozhi, T. (2013). Automatic segmentation of brain tumor using K- Means clustering and its area calculation. International Journal of advanced electrical and Electronics Engineering, 2(2), 130-134.

4. Elavarasan A. "Fourier Transform Infrared, X-Ray Diffraction and Energy Dispersive X-Ray Analysis Studies on the Adsorption of Copper Ii Ions Prepared From Mimusops Elengi Leaves in Aqueous Solution against Acid Activated Carbon". International Research Journal on Advanced Science Hub, 2, 8, 2020, 52-56. doi: 10.47392/irjash.2020.93

5. R Soundararajan; V Hariprasath; R Keerthivasan; S Muthukumar; C Naveenkumar. "Experimental Investigation of EDM Process Parameters on Aluminum Nano composite using Taguchi Technique". International Research Journal on Advanced Science Hub, 2, 5, 2020, 1-7. doi: 10.47392/irjash.2020.25

6. J. G. A. Barbedo, A novel algorithm for semi-automatic segmentation of plant leaf disease symptoms using digital image processing. "Tropical Plant Pathology", 41(4), 210-224,2016.

7. K. Bhargavi and S. Jyothi, A Survey on Threshold Based Segmentation Technique in Image Processing. International Journal of Innovative Research and Development|| ISSN 2278-0211, 3(12), 2014.

8. C. Caballero and M. C. Aranda, Plant species identification using leaf image retrieval. In Proceedings of the ACM International Conference on Image and Video Retrieval (pp.327-334). ACM, July. 2010.

9. K. D. Kharat, V. J. Pawar and S. R. Pardeshi, "Feature extraction and selection from MRI images for the brain tumour classification", IEEE International Conference on Communication and Electronics Systems (ICCES), pp. 1-5, 2016.

10. G. Cerutti, L. Tougne, J. Mille, A. Vacavant, and D. Coquin,Understanding leaves in natural images-a model-based approach for tree species identification. Computer Vision and Image Understanding, 117(10), 1482-1501, 2013.

11. J. Chaki and R. Parekh, Plant leaf recognition using shape based features and neural network classifiers. International Journal of Advanced Computer Science and Applications,2(10), 2011.

12. S. B. Dhaygude and N. P. Kumbhar, Agricultural plant leaf disease detection using image processing. International Journal of Advanced Research in Electrical, Electronics and Instrumentation Engineering, 2(1), 599-602, 2013. 